\section{Early intervention with children with Down syndrome - past and future issues}

\section{Claes Nilholm}

\author{
Department of Communication Studies \\ Linkoping University \\ Sweden
}

Firstly, research addressing early intervention in Down syndrome is discussed. It is argued, in contrast to prior reviewers, that early intervention in Down syndrome does seem to yield positive effects in different developmental domains. However, the evidence of long-term effects appears to be mixed, albeit the implications of this state of affairs are arguable. Secondly, some recent trends in early intervention research are outlined. The implications of the recent emphasis on pinpointing strengths and weaknesses in Down syndrome and the emergent recognition of the importance of the context of child development are spelled out. The consequences of a contextualized approach to child development is discussed particularly in relation to the notions of outcome variables and the wider context of development, i.e. in terms of the impact of early intervention on families and the long-term goals of early intervention. Finally, it is argued that the time seems ripe to situate the early intervention movement in its sociocultural context, i.e. in the nexus of political, ideological and scientific factors.

(c) 1993, 1999. The Down Syndrome Educational Trust Down Syndrome Research and Practice 19964 (2) $51-58$
Introduction

The purpose of the present paper is twofold. Firstly, reviews of research into early intervention with children with Down syndrome are discussed in order to summarize the conclusions that can be drawn from the field of early intervention with children with Down syndrome. Secondly, the enterprise of early intervention, and the evaluation of early intervention efforts, with children with Down syndrome is discussed from the perspective of some recent theoretical reorientation in the early intervention movement at large.

\section{Reviews of early intervention with children with Down syndrome}

Being identifiable at birth, Down syndrome has appeared as a suitable target for early efforts to ameliorate psychological and linguistic development. Since the 1970s, several reports have published outcomes of intervention specifically aimed at this group. These evaluation studies are, to varying degrees, accompanied by methodological problems regarding such factors as the selection of participants, the use of controls and the account of program content as well as the relation between the content and the initiation of the program, the choice of statistical analysis, the choice of outcome variables and tests (cf. Gunn and Berry, 1989). An additional problem pertains to the measurement of effects at the group level, which assumes equal effects on participants (Dunst, 1986).

The early intervention literature concerning Down syndrome has been reviewed on several occasions (Gibson and Fields, 1984; Carr, 1985; Foreman and Ward, 1986; Casto, 1988; Gibson and Harris, 1988; Gunn and Berry, 1989 and Spiker, 1990). Some methodological problems pervade this second-order literature as well, e.g. as regards the selection of studies. Gibson and Harris (1988) reviewed 21 studies, i.e. all those available in the peer-reviewed research literature at the time, while Casto (1988) selected 16 studies, the ones that were "found", for meta-analysis. Only 9 of these refer to studies analyzed by Gibson and Harris (1988). A further complication concerns what weight of evidence will be given to a particular study. While all the reviewers are well aware of the methodological problems inherent in this literature, no attempt is made to rank the studies in terms of credibility. Indeed, this seems to be an impossible task. How would one evaluate the effect of a methodological problem such as a specific biased selection of participants, the effect of a particular theory of intervention, the implementation of this theory into practice or the use of involved testers? Most reviewers seem to settle for this, arguing that while early intervention in Down syndrome does seem to yield measurable results, at least in the short term, further conclusions regarding effectiveness seem to be premature (Carr, 1985; Foreman and Ward, 1986; Casto, 1988 and Spiker, 1990). However, Gibson and Harris (1988) believe there are grounds for such additional conclusions, arguing that the evidence suggests that there is:

"...consistency of short-term benefits in the growth of finer motor skills, simple social repertoire and $D Q / I Q$ scores, but conflicting evidence in support or not of benefits in the gross motor, linguistic and cognitive/ academic domains" (Gibson and Harris, p. 1). 
Further, Gibson and Harris (1988) claim that follow-up studies of the early school years exhibit disappointing outcomes. Thus, since Gibson on several occasions has made analyses of the state of the art of early intervention in Down syndrome (Gibson and Fields, 1984; Gibson and Harris, 1988 and Gibson 1991a), it does seem worthwhile to scrutinize the line of argumentation leading to this conclusion, and this mainly for two reasons. The notion that training improves performance in some developmental domains, but not in others, is of theoretical significance in a developmental perspective. This would imply that either developmental domains not amenable to training are solidly genetically programmed, or that the intervention programs are poorly conceptualized. In a similar vein, it is counterintuitive, as expressed in such notions as "practice makes perfect", i.e. it is a deeply held cultural assumption that most behaviors are amenable to instruction. For these reasons, the review by Gibson and Harris (1988) will be discussed at length in order to examine whether their conclusions are warranted.

Gibson and Harris (1988) pooled 21 studies of early intervention in Down syndrome, i.e. all those available in the peer-reviewed research literature at the time. A rough estimate from the figures presented suggests that interventions up to the age of 6 and for at least five weeks qualified as early interventions. For each program, a number of variables were listed: number of subjects, chronological age at program entry, type of control, type of evaluation measures, duration of intervention, project orientation and "deliver" system. For each study, the targeted developmental domains were documented. The total set of domains were: general motor status, eye-hand/finer coordination, socialization status, speech/language, DQ/IQ/MA and cognitive/academic facility. Further, the developmental domains targeted in a particular study were divided into two categories, "most growth domain" and "least/no growth domain". In this way, no distinction was made between a growth domain where a relatively small, as compared to other domains of the particular study, but statistically significant effect, was reported, and domains where no effect, in statistical terms, was discernible. Thus, sometimes a statistically significant effect of a domain was classified as "least/no growth domain". In the result section, the category earlier referred to as "least/no growth domain" becomes identified as "little or no improvement". This semantic glide suggests a reframing of the earlier relativistic notion. Finally, longevity of treatment effect for domains were shown for the few studies that reported such effects.

Thus, what is the status of the conflicting evidence concerning treatment effects in the speech/language, cognitive/academic and the gross-motor domains and of the documentation of the potential lack of long-term effects? Since it is difficult to discern which four studies Gibson and Harris (1988) assign to the cognitive/academic domain, this domain will be left out in the following discussion. The other issues will be discussed in turn.

\section{Speech and language}

Beginning with the speech/language domain, six studies "claim favourable outcomes and four find little or no improvement" (Gibson and Harris, 1988). Studies by Brinkworth (1973), Hayden \& Dmitriev (1975) and Aronsson \& Fällström (1977) are classified as showing "little or no improvement" for the speech/lanquage domain. In the
Brinkworth study (1973), the experimental group received a mean of 99.9, and the control group a mean of 69.7 on the hearing/speech scale of the Griffith developmental scales. The difference was statistically significant. Considering the $\mathrm{t}$-values, in contrast to the means, the difference in the hearing/speech measure is the second largest of the five domains investigated. In the Aronsson and Fällström (1977) study, the training group showed an average increase of 7.9 months, as compared to 3.7 months for the control group, on the Griffith hearing/speech scale during the 18 months of training. No tests of significance are provided. However, in the absence of such a test, the reasonable conclusion would be that the training had a positive effect in the hearing/ speech domain. Further, in both these studies, the differences on the hearing/speech scale were the largest in the follow-up testing. Hayden and Dmitriev (1975) present different sets of data on language development. Gibson and Harris (1988) choose to present the figure of 39-100 competency per age level in the early preschool section of the intervention program. These figures are, of course, hard to interpret. However, in Hayden's and Dmitriev's paper (1975), another comparison is made between 14 preschool children, who had attended preschool for six to twelve months with five children who had just entered the program. The developmental lag on the Peabody Picture Vocabulary Test was 6.4 months for the former group and 21 months for the latter. Consequently, the conclusion of "little or no improvement" does not seem reasonable although, admittedly, it is hard to draw any conclusion from these data. Following the line of reasoning by Gibson and Harris (1988), the most reasonable conclusion seems to be that there is hardly any evidence against the hypothesis that early intervention programs are beneficial as regards the language/speech domain.

\section{The gross motor domain}

A similar line of reasoning applies to the interpretations of the outcome of studies addressing the gross motor domain. According to Gibson and Harris (1988), only three studies out of nine suggest that training effects in the gross motor domain are reliable. The six studies classified as reporting "little or no improvement" seem to be Brinkworth (1973), Bidder, Bryant and Gray (1975), Hanson and Schwarz (1978), Hayden and Dimitriev (1975), Clunies-Ross (1979) and Piper and Pless (1980). In the Hanson and Schwarz (1978) study, taken as an indication of "little or no improvement in the gross motor domain", median ages of sitting supported with head steady, rolling over independently and walking with support for the experimental group are $4.25,4.38$ and 13 months, compared to the agenorms of 5,7 and 20 months (no tests of significance provided). Similarly, Clunies-Ross (1979) reports progressively higher developmental indexes (no statistical testing provided) in the gross motor domain with time in the program. Two studies, Bidder et. al. (1975) and Brinkworth (1973) report non-significant developmental advantages, as measured by the locomotor scale of the Griffith test, for the experimental group after six months of training. The outcome reported by Hayden and Dimitriev (1975) of 30100 competence per age level is presented and, as discussed earlier, best left uninterpreted. Finally, the study by Piper and Pless reports a larger decline for the experimental group. Thus, of six studies taken by Gibson and Harris (1988) as indication of "little or no improvement", two seem to involve quite large gains by the treatmentgroup, two involve gains, although insignificant, one does 
not have a bearing on the issue at stake and one is a clear indication of no improvement. Even though the pattern is not as clear as to the hearing/speech domain, it does seem that the bulk of evidence suggests positive effects for gross motor training.

\section{Long-term effects}

An additional aspect of early intervention programs discussed by Gibson and Harris (1988) are the long-term benefits of early intervention. The reviewers report such data from eight studies (Brinkworth, 1973; Aronsson and Fällström, 1977; Ludlow and Allen, 1979; Conolly, Morgan, Russel and Richardson, 1980; Hudson and Clunies-Ross, 1984; Pieterse and Center, 1984, and Cunningham, 1987). The studies by Brinkworth (1973), Aronsson and Fällström (1977), Ludlow and Allen (1979) and Cunningham (1987) are taken as evidence of loss of gains at follow-up testing. While the Brinkworth (1973) and the Cunningham (1987) studies point in this direction, the Ludlow and Allen (1979) study exhibits a narrowing of differences in IQ scores, but no such leveling for school placement. In the Aronsson and Fällström study involving eight pairs of children with Down syndrome matched for chronological age, several changes took place between the intervention and the follow-up study. One pair of children was excluded, and 8 of the remaining 14 children had been moved to other institutions. Consequently, it is hard to draw any conclusions from this study with respect to long-term effects since we do not know what effects these new environments had on the development of the children. The study by Conolly et. al. (1980) supports long-term effects of early intervention. The evidence appears to be mixed. However, what would the conclusion be if long-terms effects are not substantiated ? No early intervention or continued intervention? Examples of such continuous intervention are characteristics of the intervention programs reported by Hudson and CluniesRoss (1984) and Pieterse and Center (1984), especially the latter. Gibson and Harris (1988) do not discuss the outcome of these studies with regard to some kind of control condition; that is, some kind of achievement of a comparable group of children with Down syndrome. Instead, the comparison is now made with non-Down syndrome "comparison" children. The argument turns from being concerned with effects of early intervention to possibilities of school integration. However, the papers are not informative about long-term effects, understood as a comparison between treated and untreated groups of children with Down syndrome, at all.

To sum up, it does seem that early intervention in children with Down syndrome, given the methodological shortcomings of the field, provides support for reliable effects across different developmental domains. However, in agreement with Gibson and Harris (1988), the question of long-term effects does seem to be an open issue. Even though the effectiveness of early intervention is still debated, it has become an accepted practice. While it is questionable if "early" is better in early intervention with children with Down syndrome (Casto, 1988; Gibson and Harris, 1988), "early" is accepted as better. One cannot on an a priori basis reject the hypothesis that early intervention is less based on scientific knowledge, than on deeply held cultural beliefs. Maybe the time is ripe to broaden the scientific interest in order to investigate the interplay of such beliefs with how early intervention is conceived. As noted by Marfo and Cook (1991), the "efficacy" issues seem to be a typical American preoccupation and earlv intervention can be considered legitimate on other grounds, such as in terms of humanistic values. As the "efficacy" issue has been tied to economical resources, the quest to prove the efficacy of early intervention programs has become vital. However, in this way, more important research concerns might have become neglected, such as more specific questions concerning training methods. While the historical process seems to make the control-group design anachronistic, methods addressing the differential effectiveness of training methods do seem suitable, both from ethical and scientific standpoints.

\section{Recent trends in early intervention - implications for early intervention with children with Down syndrome}

The early intervention movement is heading towards an emphasis on the context of the child's development Particularly, works by Sameroff and Chandler (1975) and Bronfenbrenner (1979) have been influential in this regard. In a sense, the unit of analysis proposed is not the child and his/her characteristics as an isolated individual, but rather "the child in context", i.e. the child as he/she appears in his/her interactions with the environment. Consequently, the prior significance given to skill training has in part been replaced by a focus on family and on interaction (Guralnick and Bennett,1987, and Marfo and Cook, 1991), i.e. the target of intervention is no longer taken to be the child as such but rather the child-environment context. Moreover, suggestions for the assessment of a new set of outcome variables have been put forward. Especially, the need for measures of family functioning, as well as measures of child development not exclusively dealing with cognitive development, have been suggested. This is in line with Zigler's (1990) proposal that other aspects than cognitive ones regarding the development of the child are important. Hence, we can discern a trend towards viewing the child as a multifaceted active participant developing in an ever-changing environment. Also, the fact that the child is a participant in multiple contexts has become increasingly recognized (Sameroff, 1992). Additionally, the need to pinpoint the characteristics of different groups of children, as well as the characteristics of the individual child and her/his family, is becoming increasingly acknowledged (Gibson, 1991b, Marfo and Cook, 1991). In this way, the future prospect of the early intervention movement is sometimes pictured as one in which increasingly refined, theoretically grounded techniques will improve the future prospects of the child targeted for early intervention.

I will discuss these recent trends in early intervention and their implications for intervention, and the evaluation of interventions, with children with Down syndrome. Firstly, I will argue that it becomes problematic, given a contextual view of development, to pinpoint the relative strengths and weaknesses of Down syndrome children as a group, and as individual children, in order to design specific interventions for the group, and for the individual child. Secondly, two aspects of a contextual approach will be discussed pertaining to the issue of what outcome variables to study; how context specific an outcome measure should be, and the additional issue of the responsibility of families and professionals in the development of the child. Thirdly, the full implications of viewing the child as a part not only of family and family-professional systems, but the embeddedness of these contexts in the wider social and 
ideological context, and the related issue of where change is to occur, will be discussed.

\section{Early intervention and strengths and weaknesses in children with Down syndrome}

The notion of adapting intervention programs to the specific characteristics of children with Down syndrome as a group, as well as to the particular child with Down syndrome, raises the issue of what these characteristics are, i.e. how do we know in what areas children with Down syndrome are different from normal children, or children with other forms of learning disability? Secondly, once mapped out, what are the consequences of early intervention if these areas could be mapped out? Apart from the well-known problems for children with Down syndrome with motor and language development, are there additional insights from controlled studies that can be used in early intervention programs? Even though an early intervention approach which addresses the special characteristics of children does seem logical, precautions should be made. Particularly two things will be discussed for the present purposes; 1) The problem of proving a qualitative difference in development, and, 2) The problem of interpreting a difference in order to recontextualize a controlled finding into an early intervention program.

There are problems in pinpointing qualitative differences in development for children with Down syndrome, apart from the general developmental lag, i.e. to prove qualitative differences in the development of children with Down syndrome matched for mental age with normal children or children with other forms of developmental disability. Wagner, Ganiban and Cicchetti (1991) discuss this problem in relation to infant development. In this context, The Bayley scales of infant development (1969) has been used extensively for matching purposes.

However, as argued by Wagner et. al. (op. cit.), it is doubtful what such matching means, since these scales, on the one hand, are weakly correlated with cognitive measures in childhood and, on the other, contaminate mental and motor abilities. Thus, if the outcome variable is dependent on motor abilities, e.g. in investigations of exploratory behaviour, it seems likely that, on a theoretical basis, MAmatching on the Bayley scales would involve children with Down syndrome who are ahead in mental development and behind in motor development. Thus, the outcome of such an investigation would be hard to interpret. Most importantly, and this line of reasoning will apply also to older children with Down syndrome, as well:

... to support a "Difference" claim , individuals must perform differently better or worse as a function of some manipulated variable (e.g. auditory vs. visual presentation, stimulus complexity, etc.). In other words, to postulate that a specific factor such as mental retardation or Down syndrome affects task performance, one must first demonstrate that an interaction between the factor and the stimulus condition exists. (p. 171.).
Thus, any difference in e.g. a cognitive task could be attributable to several factors; e.g. temperament differences, motivation, familiarity with the task materials and motor abilities. Wagner et. al. (op. cit.), in their review of attention, memory and perception in Down syndrome infants, conclude that:

...we have yet to see a study that implicates a qualitative difference in specifically cognitive mechanisms on the part of the infant with Down syndrome (p. 172).

Thus, for infants with Down syndrome, it does seem problematic to point out specific cognitive weaknesses and similar problems emerge for older children. E.g. the list provided by Gibson (1991b) of weaknesses in Down syndrome involves several factors that would need much more research in order to disentangle their mutual interdependencies. Gibson (op. cit.) points out, among other factors, verbal memory, impulsive responding, poor motivation and limited coding for verbal material, as particular weaknesses in Down syndrome. It is not clear how these different factors could be disentangled either theoretically or empirically.

Secondly, and more importantly, given a transactional approach to development, the child's behavioural repertoire at a certain moment in its development would be a function of its interaction with the environment. Thus, every difference between a group of children with Down syndrome and normal children, or children with other mental disabilities, cannot, by definition, be accounted for by Down syndrome itself. In fact, Down syndrome does not explain anything, since the group investigated comprises children with Down syndrome who have had particular transactions with their environments. Here, transactions should be understood in a wide sense, thus encompassing stimulation in the environment as well as health care factors. In this perspective, controlled studies of children with Down syndrome imply the occurrence of a universal child with Down syndrome, i.e. a child whose characteristics could be mapped out separated from the prior transactions of the child with her/his environment.

To sum up, pinpointing particular weaknesses in Down syndrome, albeit a reasonable standpoint, does seem to need further elaboration if it is to have any bearing on early intervention programs, i.e. apart from the well-known common problems in Down syndrome such as hypotonia, speech and motor development, which are common enough to surface in different types of environments. Three criteria should have to be fulfilled in order to inform early intervention practices: 1) The weakness should be wellestablished in the sense described, 2) It should be meaningfully related to subsequent development of important behaviors or be of significance in itself, 3) Methods of training should be devised, which enhance the skill in question. Since controlled studies have usually been devised in order to focus on group differences, individual differences would have to be addressed too in order to strengthen the validity of such studies.

\section{Outcome variables}

The importance of investigating behaviour in context has implications for what, as well as how context-specific, the outcome measures should be (cf. Granlund, Björk-Åkesson, 
Brodin and Olsson, 1995). On the one extreme, normative measures supply the outcome measures. On the other extreme, the outcomes are a part of the child's context itself, i.e. the child's development, and the impact of intervention efforts, are evaluated in terms of how the child's development in the particular contexts of the family or the preschool group is progressing. Both these approaches can be combined with focuses of attention that are selected by parents or professionals or negotiated between these two groups, e.g. adaptation (as measured by tests, or by "real-life" adaptation), intellectual development (as measured by IQ tests, or by the intellectual demands of the child's environment, e.g. the preschool or the family). I suggest that taking a contextual approach seriously involves evaluation of the outcomes of intervention efforts in terms of the child's functioning in his/her environment. Intervention efforts and their evaluation have to consider the demands made on the child in his/her immediate contexts. Such goals would, given a contextualized approach to child development, be expressed in behaviors that are functional in these very contexts. In this regard, IQ-measures or language test performance are too general. E.g. the language skills of a child with Down syndrome is presumably important for his/her management of the context of a kindergarten. However, rather than the child's test performance, pragmatic abilities, i.e. the skills in negotiating relations, playing and the like, and understanding the rule system of this micro-context are what become important. The utilization of norm-referenced tests is informative to the extent that they mirror such issues.

The proposal of a new set of outcome variables in early intervention research could be interpreted as a larger sensitivity to the needs of the family. In a recent study of early intervention with a group of children with Down syndrome, and two additional disability groups (Shonkoff, Hauser-Cram, Wyngaarden Krauss and Upshur, 1992), several measures of child characteristics, service measures (both within and outside early intervention programs) and family variables were analyzed. The major findings of this study were the variation between children, the adaptability of families, the multifaceted character of early intervention practices and of correlates of change. However, given the correlational character of the data, the meaning of the findings are not easy to interpret (see Sameroff, 1992, for an elaborated discussion). The most interesting finding is perhaps that these relatively well-off families with a disabled child seemed to fare quite well, being firmly convinced of the desirability of raising their children. However, we do not hear "the voice" of the family in this report. A longer trend in the upbringing of children with learning disabilities can be discerned, in which the responsibility of the family seems to have been given greater importance. Thus, a movement from de-institutionalization, to professionally-driven training regimes, to proposals about family-centred intervention can be seen as a changing balance between the community and the family. In a sense, early intervention implies a socialization practice towards the family. The birth of a disabled child most often constitutes a fundamental redefinition of the self-perception of the family, now becoming "a family with a disabled child". More knowledge is needed about this socialization process and the role professionals play in it. So far, the research on family involvement is mostly carried out according to normative models, e.g. whether families are involved in goal-setting and so forth. For example, in the study by Shonkoff et. al.
(1992), the outcome variables pertaining to family adaptation were mother-child interaction, parenting stress and social support. While such factors are important, the encounter of families with a professional community also implies a cultural meeting in its own right. Such transactions between family environments and professionals milieus need to be studied separated from the professional's interest. As Gunn and Berry (1989) suggest:

\begin{abstract}
... professionals tend to divorce themselves from the social ecology of early intervention. There may be so much emphasis on the skills which have to be taught to others that the professionals fail to examine their own role and influence. What are their own attitudes to disability and what are their expectations of development for the child? (p. 240).
\end{abstract}

Even if professionals take the social ecology into consideration, it does seem unlikely that the evaluation of the impact of early intervention on the family setting, could be accomplished from the standpoint of the professional perspective. Thus, given the multi-disciplinary approach of early intervention, involving medical, psychological and educational experts, it does seem desirable to widen the scope of attention into the areas of sociology and anthropology. In a study of this kind concerning genetic counselling, Rapp (1988) focused on the multiple conceptions that arose when professional and family spheres meet in the public arena. In Rapp's approach, the meaning that families construe in their meetings with professionals are seen as something which has to be studied in terms of the perspective of the families. The issue at stake is who is going to define the quality of life for a particular child. In this regard, the joint effort by professionals and parents, as well as the tensions in their perspectives, needs to be addressed.

\section{The context of early intervention}

How much context is then to be written into evaluations of early intervention? The importance of this issue might become clearer in reconsidering the study by Aronsson and Fällström (1977), which is praised as one of the few early intervention studies which has used a control group and a long-term follow up. This study was conducted in a Swedish nursing home for mentally disabled children, Sister Ester's home in Lödöse, Sweden. In the 1970s, the wellknown Swedish administrator of mental retardation services, Karl Grunewald, made an inventory of the children with Down syndrome living in this institution:

\begin{abstract}
... in 14 of the 17 cases the motives were insufficient for a nursing home placement. The most noteworthy outcome of the inventory was that the parents had made the decisions about nursing home placement as early as a few months after the birth of the child. The profound and decisively important decision to leave ones child to societal care was made by the parents without receiving qualified help to work at their problem (Graninger and Lovén, 1994 , p. 58f/ my translation).
\end{abstract}

Thus, in considering the total context of the child's development, the outcome of the Aronsson and Fällström study would have to be revised. For every child, the 
importance of growing up in a family is accepted nowadays and is considered to be of fundamental importance in terms of the child's quality of life. In a similar vein, the interpretation by Gibson and Harris (1988) of the study by Pieterse and Center (1984) makes the important point about the wider context of child development especially clear. This study involved eight children with Down syndrome, who had been in early intervention programs for several years and who had been mainstreamed in regular schools. At the time of the study, the children were in kindergarten through third grade. The authors are optimistic as regards the outcome of this study. Especially, measures of social adjustment and the attitudes of parents, school officials and teachers contribute to this. On the other hand, available outcomes regarding reading achievement show that only one child (grade 1) scores above the 20th percentile for word knowledge and one (grade 2) for comprehension on normed tests. For arithmetic tests, no child scores above the 5th percentile on the normed test. Pieterse and Center conclude:

This suggests that with structured early intervention and itinerant teacher assistance, selected Down's syndrome children function within the range tolerated in regular classrooms, at least in reading achievement (Pieterse and Center, 1984, p. 14).

It is illuminating to compare this conclusion with the one drawn by Gibson and Harris (1988) from the same study and an additional one with similar outcomes by Hudson and Clunies-Ross (1984):

Hence, the paradox of DS children who meet with initial peer and teacher acceptance but who face future personal-social rejection as cognitive development fails to keep pace with the increasing conceptual demands of academic curricula and the growing subtleties of the social interaction process. (P. 11.)

Of course, we do not know if the speculation about the future development of mainstreamed children with Down syndrome will hold true. What does seem likely is that children with Down syndrome, as a group, will face several problems as regards academic achievement. For example, the average IQ of the selected group of children with Down syndrome in the Pieterse and Center (1984) study is 60 (range 48-70), which is comparable with the outcome of several follow-up studies summarized by Rynders and Horrobin (1990), (with projects involving younger children reporting higher scores). These outcomes raise two related issues; 1 ) what are the contexts that children with Down syndrome are socialized into, and, 2) what aspects of the child-environment relation is to be changed. Given the intellectual potentialities of these children, an integration of these children would by necessity be related to change in the practice of schooling:

The change to more formal methods of instruction may well bring additional problems of which we are at present unaware. However, on the basis of our current experience we would claim that if a policy of integration into regular infant's primary classes is to be of benefit to disabled children. normal peers. involved parents and regular schools, it should be preceded by systematic and effective early intervention, implemented by an informed and committed school executive and staff and accompanied by the provision of specialist support from the point of school entry, as well as the maintenance of longitudinal, ideographic data (Pieterse and Center, 1984, p. 19).

However, it does seem likely that the future of these children would be dependent on a change in the method of school work. Since child socialization always involves preparation for the future, it would seem reasonable that such future goals would be spelled out for early intervention programs. Conceptions such as "least restrictive environment", rather than taken as a goal, should be further operationalized. The unproved assumption seems to be that it is better for the child to be mainstreamed as long as possible.

The Pieterse and Center (1984) study also accentuates the second issue raised above. Notably, a part of the study, and the outcome of the study, seem to have been related to the adaptation of the children's immediate school context in terms of involving the school staff and providing support from an itinerant school teacher. Thus, by adjusting the school context to the needs of the children, successful integration was achieved. In a perspective in which behaviour by definition is related to contexts of development, the structure of these contexts themselves become targets of intervention. Expanding the notion of context, and changing contexts, the question can be put: in what sense can contexts be changed in order to enhance the potentialities of children with Down syndrome?

\section{Concluding remarks}

The emerging view of the disabled child as an active participant in multiple changing contexts seem to be a theoretically interesting approach. The implications of such an approach for early intervention and evaluation of early intervention with children with Down syndrome seem to have far-reaching consequences. As regards the practice of early intervention, it has been argued that a theoretical perspective, which takes the notion of context seriously, makes the recontextualization of findings of controlled studies appear to be problematic with regard to their implications for early intervention. In regard to the evaluation of early intervention, it has been argued that a contextualized version of outcome measures is needed and that the family-professional relationship should be investigated from new standpoints. Also, both with regard to the practice and evaluation of early intervention, the need to explicate longer term goals has been proposed. The enlarged notion of context raises important methodological questions:

When context was defined as maternal practice, it was relatively easy to bring a mother and child into the laboratory and get the mother to vary her behaviour in order to study the effects on the child. With context now defined as the micro-, meso-, exo- and macrosphere of all social institutions from the most proximal to the most distal, it is impossible to use traditional research models (Sameroff, 1992, p.155). 
So what methods are there to use? I suggest that there is a need for two different types of studies. Given the complexity of variables affecting child development, and the processual nature of these relationships, there is a need for studies of early intervention that supply detailed, descriptive accounts of these complexities, preferably on a case basis (cf. Emde and Brown (1978)). Accounts of how particular children with Down syndrome, given their individual prerequisites for development, are received and develop within particular contexts seems much needed. If the unit of analysis is childenvironment contexts, the methodology must be adapted to this aim. However, traditional scientific rigour still has its place. Abandoning the global efficacy question will perhaps provide room for more interesting "local efficacy questions "such as what transactions are most beneficial in reaching a certain goal in the child's context of development. Thus, instead of evaluating early intervention on a large-scale basis, with methodological shortcomings of an often "practical" nature, it seems that strictly designed studies of methods of intervention could yield valuable insights.

Finally, the time seems ripe to illuminate the workings of macro-factors in the context of early intervention. Especially, the interplay of ideology, science and early intervention do seem to need further elaboration. For example, in what sense have scientific findings, as opposed to beliefs of educationalists, influenced the practice of early intervention, and to what extent have extra-scientific factors, such as changes in ideology and political concerns connected with the developmentally disabled done so ? This, and related questions, need to be addressed.

\section{References}

Aronsson, M. and Fällström, K. (1977). Immediate and long-term effects of developmental training in children with Down's syndrome. Developmental Medicine and Child Neurology, 19, 1977

Bayley, N. (1969). The Bayley scales of infant development. New York: Psychological Corporation. Bidder, R.T., Bryant, G. and Gray, O.P. (1975). Benefits to Down's syndrome children through training their mothers. Archives of Diseases in Childhood, 50, 383-386.

Brinkworth, R. (1973). The unfinished child. Effects of early home training on the mongol infant. In A.D.B. Clarke and A.M. Clarke (Eds.) Mental retardation and behavioural research. Edinburgh and London: Churchill Livingstone.

Bronfenbrenner, U. (1979). The ecology of human development-experiments by nature and design. Cambridge, Ma: Harvard University Press.

Carr, J. (1985). The development of intelligence. In D. Lane och B. Stratford (Eds.) Current approaches to Down's syndrome. London: Cassel Educational Limited.

Casto, G. (1988). Early intervention for infants and preschoolers with Down syndrome: a review of best practices and effectiveness data. In C. Tingey (Ed.) Down syndrome: a resource handbook. Austin TX: Pro-Ed.

Clunies-Ross , G. (1979). Accelerating the development of Down's syndrome infants and young children. The Journal of Special Education, 13, 169-177.

Conolly, B., Morgan, S., Russel, F.F. and Richardson, B. (1980). Early intervention with Down syndrome children. Physical Therapy, 60, 1405-1408.

Cunningham, C. (1987). Early intervention in Down's syndrome. In G. Hosking and G. Murphy (Eds.) Prevention of mental handicap: a world view. Royal Society of Medicine Services International Conaress and Svmposium Series
No. 112, published by Royal Society of Medicine Services Limited.

Dunst, C.J. (1986). Overview of the efficacy of early intervention programs. In L. Brickman and D.L. Weatherford (Eds.) Evaluating early intervention programs for severely handicapped children and their families. Austin TX: Pro-ed. Emde, R.N. and Brown, C. (1978). Adaptation to the birth of Down syndrome infants. Journal of American Academy for Child Psychiatry, 17, 299-323.

Foreman, P.J. and Ward, J. (1986). Treatment approaches in Down's syndrome: a review. Australia and New Zealand Journal of Developmental Disabilities, 12, 111-121.

Gibson, D. (1991a). Down syndrome and cognitive enhancement: not like the others. In K. Marfo (Ed.) Early intervention in transition: current perspectives on programs for handicapped children (pp. 3-40). New York: Praeger Publishers.

Gibson, D. (1991b). Searching for a life-span psychobiology of Down syndrome: advancing educational and behavioural management strategies. International journal of disability, development and education, 38, 71-89.

Gibson, D. and Fields, D.L. (1984). Early infant stimulation programs for children with Down's syndrome: a review of effectiveness. In M.L. Wolraich and D.K. Roth (Eds.) Advances in Developmental and Behavioral Pediatrics (Vol. 5). Greenwich, Connecticut: JAI Press.

Gibson, D. and Harris, A. (1988). Aggregated early intervention effects for Down's syndrome persons: patterning and longevity of benefits. Journal of mental deficiency research, 32, 1-17.

Granlund, M., Björk-Åkesson, E., Brodin, J. och Olsson, C. (1995). Communication intervention for persons with profound disabilities: a Swedish perspective. Augmentative and alternative communication, 11, p. 49-59.

Graninger, G. and Lovén, J. (1994). 40 år med utvecklingsstörda-samtal med Karl Grunewald (40 years with the mentally retarded-conversations with Karl Grunewald). Moheda: Fontes.

Gunn, P. and Berry, P. (1989). Education of infants with Down syndrome. European Journal of Psychology of Education., 4, 235-246.

Guralnick, M.J. and Bennet, F.C. (1987). A framework for early intervention. In M.J. Guralnick and F.C Bennet (Eds.) The effectiveness of early intervention for at-risk and handicapped children (pp. 3-29). Orlando: Academic Press. Hanson, M.J. and Schwarz, R.H. (1978). Results of a longitudinal intervention program for Down's syndrome infants and their families. Education and Training of the Mentally Retarded, 13, 403-407.

Hayden, A.H. and Dmitriev, V. (1975). The multidisciplinary preschool program for Down's syndrome children at the University of Washington model preschool center. In B.Z. Friedlander, G.M. Sterrit and G.E. Kirk (Eds.) Exceptional Infant: Assessment and intervention (Vol. 3) (pp. 193-221). New York: Brunner/Mazel.

Hodapp, R.M. and Zigler, E. (1990). Applying the developmental perspective to individuals with Down syndrome. In D. Cicchetti and M. Becghly (Eds.) Children with Down Syndrome: a developmental perspective (pp. 128). Cambridge: Cambridge University Press.

Hudson, A. and Clunies-Ross, G. (1984). A study of the integration of children with intellectual handicaps into regular schools. Australian and New Zealand Journal of Developmental Disabilities, 10, 165-176.

Ludlow, J.R. and Allen, L.M. (1979). The effects of early intervention and pre-school stimulus on the development 
of the Down's syndrome child. Journal of Mental Deficiency Research, 23, 29-44.

Marfo, K. and Cook, C. (1991). Overview of trends and issues in early intervention theory and research. In $\mathrm{K}$. Marfo (Ed.); Early intervention in transition: current perspectives on programs for handicapped children (pp. 340). New York: Praeger Publishers.

Pieterse, M. and Center, Y. (1984). The integration of eight Down's syndrome children into regular schools. Australian and New Zealand Journal of Developmental Disabilities, 10, 11-20.

Piper, M.C. and Pless, I.B. (1980). Early intervention for infants with Down syndrome: a controlled trial. Pediatrics, 65, 463-468.

Rapp, R. (1988). Chromosomes and communication: The discourse of genetic counseling. Medical Anthropology Quarterly, 2, 143-157.

Rynders. J.E. and Horrobin, M. (1990). Always trainable? Never Educable? Updating educational expectations concerning children with Down syndrome. American Journal on Mental Retardation, 95, 77-83.

Sameroff, A.J. and Chandler, M.J. (1975). Reproductive risk and the continuum of caretaking causality. In F.D. Horowitz (Ed.) Review of child development research (Vol. 4) (pp 187-244). Chicago: University of Chicago Press.

Sameroff, A.J. 1992. Systems, development and early intervention. Monographs of the society for research in child development, 57, no. 6. (Commentary on Shonkoff et al.)

Shonkoff, J.P., Hauser-Cram, P., Wyngaarden Krauss, M. and Christofk Upshur. C. (1992). Development of infants with disabilities and their families: Implications for theory and service delivery. Monographs of the society for research in child development, 57 , no. 6.

Spiker, D. (1990). Early intervention from a developmental perspective. In D. Cicchetti and M. Beeghly (Eds.) Children with Down Syndrome: a developmental perspective. (pp 424-448). Cambridge: Cambridge University Press.

Wagner, S. Ganiban, J. and Cicchetti, D. (1990). Attention, memory, and perception in infants with Down syndrome. In D. Cicchetti and M. Beeghly (Eds.) Children with Down Syndrome: a developmental perspective. (pp 147-179). Cambridge: Cambridge University Press.

\begin{tabular}{c} 
Address for correspondence: \\
Dr Claes Nilholm \\
Tema Kommunikation \\
Linkopings Universitet \\
S-58183 Linkoping \\
Sweden \\
e-mail:clani@tema.liu.se \\
\hline
\end{tabular}

